\title{
Extramedullary plasmacytoma of the thyroid, refractory to radiation therapy and treated with bortezomib
}

\author{
Subash Ghimire, MD; Swapna Talluri, MD; Amir Bista, MD; Zachary Wolfe, MD; \\ Manidhar Lekkala, MD; Ashit Sarker, MD; and Bradley Lash, MD \\ Guthrie Robert Packer Hospital, Sayre, Pennsylvania
}

$\mathrm{P}$ lasma cell neoplasms involving tissues other than the bone marrow are known as extramedullary plasmacytoma (EMP). ${ }^{1}$ EMPs mostly involve the head and neck region. ${ }^{2}$ Solitary EMP involving only the thyroid gland is very rare., ${ }^{3,4}$ Because of the limited knowledge about this condition and its rarity, its management can be challenging and is often extrapolated from plasma cell myeloma., ${ }^{5,6}$ In general, surgery or radiation are considered as front-line therapy. 3,5 EMPs usually respond well to radiotherapy with almost complete remission. No definite guidelines outlining the treatment of radio-resistant EMP of the thyroid have yet been published. Data supporting the use of chemotherapy is particularly limited. ${ }^{4,7,8}$

Here, we describe the case of a 53-year-old woman with a long history of thyroiditis who presented with rapidly worsening symptomatic thyroid enlargement. She was diagnosed with EMP of the thyroid gland that was not amenable to surgery and was refractory to radiotherapy but responded to adjuvant chemotherapy with bortezomib. This report highlights 2 unique aspects of this condition: it focuses on a rare case of EMP and, as far as we know, it reports for the first time on EMP that was resistant to radiotherapy. It also highlights the need for guidelines for the treatment of EMPs.

\section{Case presentation and summary}

A 53-year-old woman presented to the emergency department with complaints of difficulty swallowing, hoarseness, and neck pain during the previous 1 month. She had a known history of Hashimoto's thyroiditis, and an ultrasound scan of her neck 6 years previously had demonstrated diffuse thyro- megaly without discrete nodules. On presentation, the patient's vitals were stable, and a neck examination revealed a firm and enlarged thyroid without any cervical adenopathy. Laboratory investigations revealed a normal complete blood count and comprehensive metabolic panel. She had an elevated thyroid-stimulating hormone level of 13.40 $\mathrm{mIU} / \mathrm{L}$ (reference range, $0.47-4.68 \mathrm{mIU} / \mathrm{L}$ ) and normal thyroxine level of $4.5 \mathrm{pmol} / \mathrm{L}$ (reference range, 4.5-12.0 pmol/L). A computerized tomography (CT) scan of the neck revealed an enlarged thyroid gland (right lobe length, $10.3 \mathrm{~cm}$; isthmus, $2 \mathrm{~cm}$; left lobe, $8 \mathrm{~cm}$ ) with a focal area of increased echogenicity in the midpole of the left lobe measuring $9.5 \mathrm{~mm} \times 5.5 \mathrm{~mm}$. The patient was discharged to home with pain medications, and urgent followup with an otolaryngologist was arranged. A flexible laryngoscopy was done in the otolaryngology clinic, which revealed retropharyngeal bulging that correlated with the thyromegaly evident on the CT scan.

Because of the patient's significant symptoms, we decided to proceed with surgery with a clinical diagnosis of likely thyroiditis. A left subtotal thyroidectomy with extension to the superior mediastinum was performed, but a right thyroidectomy could not be done safely. On gross examination, a well-capsulated left lobe with a tan-white, lobulated, soft cut surface was seen. Microscopic examination revealed replacement of thyroid parenchyma with sheets of mature-appearing plasma cells with eccentric round nuclei, abundant eosinophilic cytoplasm without atypia, and few scattered thyroid follicles with lymphoepithelial lesions (Figure 1A). Immunohistochemistry confirmed plasma cells with

Accepted for publication July 17, 2018. Correspondence: Subash Ghimire, MD; drsubash01@gmail.com. Disclosures:

The authors report no disclosures or conflicts of interest. JCSO 2018;16(5):e206-e209. (C2018 Frontline Medical

Communications. doi: https://doi.org/10.12788/jicso.0414 


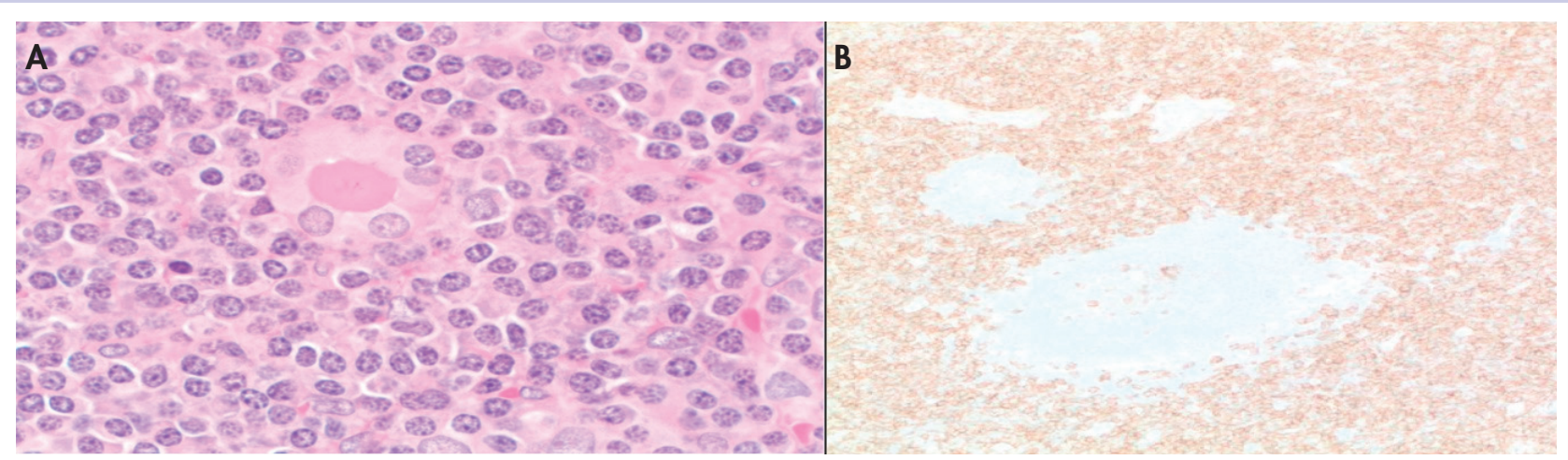

FIGURE 1 A, Mature plasma cells surrounding follicles (H\&E, ×650). B, CD138 positive plasma cells in thyroid, with unstained areas showing lymphoid follicles (immunohistochemistry, x100).
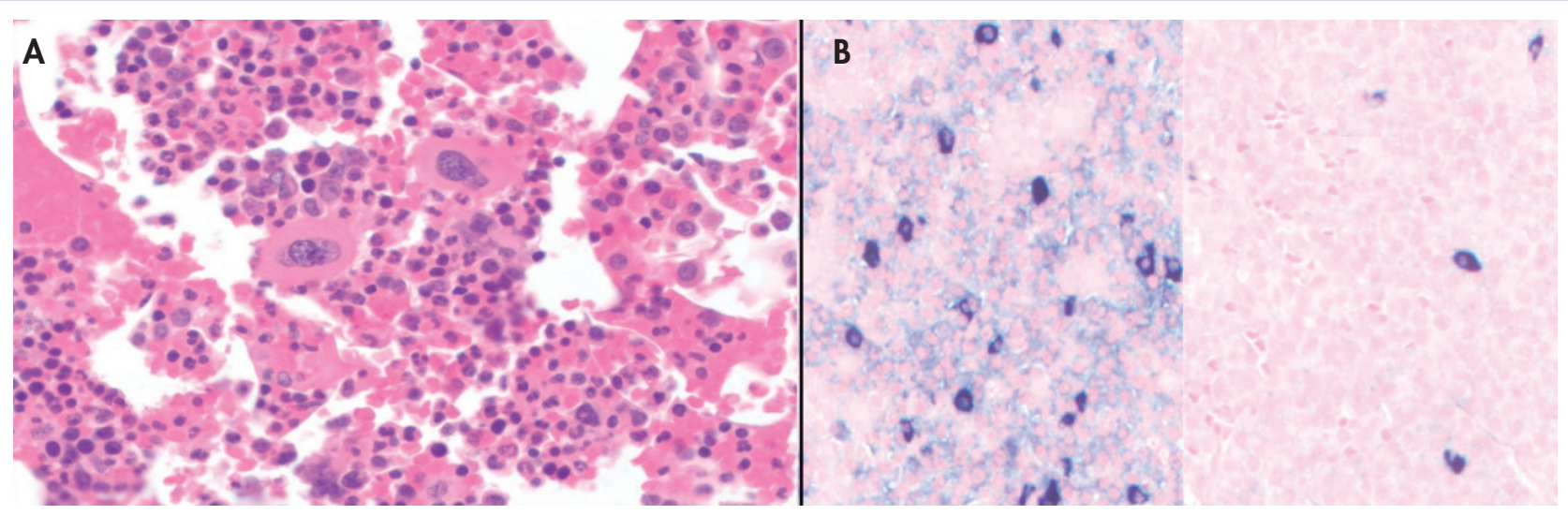

FIGURE 2 A, Trilineage hematopoesis with scattered mature-appearing plasma cells in bone marrow (H\&E, $\times 400)$. B, Proportionate distribution of kappa (left) and lambda (right) light chains in bone marrow (FISH, ×200).

expression of CD138 (Figure 1B). Fluorescence in situ hybridization (FISH) showed that the neoplastic plasma cells contained monotypic kappa immunoglobulin light chain messenger RNA. Clonal immunoglobulin gene rearrangement was detected on polymerase chain reaction. A diagnosis of plasmacytoma of the thyroid gland in a background of thyroiditis was made on the basis of the aforementioned observations.

After that diagnosis, we performed an extensive workup for plasma cell myeloma. Bone marrow biopsy showed normal maturing trilineage hematopoiesis with scattered mature-appearing plasma cells Figure 2A. Flow cytometry showed a rare $(0.2 \%)$ population of polytypic plasma cells and was confirmed by CD138 immunohistochemistry. FISH showed proportionate distribution (2-5:1) of kappa and lambda light chains in plasma cells (Figure 2B). Serum protein electrophoresis showed normal levels of serum proteins with no M spike. Serum total protein was $7.9 \mathrm{~g} /$ $\mathrm{dL}$, albumin $5.0 \mathrm{~g} / \mathrm{dL}, \alpha 1$-globulin $0.3 \mathrm{~g} / \mathrm{dL}, \alpha 2$-globulin $0.8 \mathrm{~g} / \mathrm{dL}, \beta$-globulin $0.7 \mathrm{~g} / \mathrm{dL}$, and $\gamma$-globulin $1.6 \mathrm{~g} / \mathrm{dL}$, with an albumin-globulin ratio of 1.47 . Calcium and $\beta 2$-microglobulin were also in the normal ranges. Serumfree kappa light chain was found to be elevated $(20.9 \mathrm{mg} / \mathrm{L}$; reference range, 3.3-19.4 mg/L). The immunoglobulin $\mathrm{G}$ level was also elevated at $3,104 \mathrm{mg} / \mathrm{dL}$ (reference range, $700-1,600 \mathrm{mg} / \mathrm{dL}$ ).

A positron-emission tomographic (PET) scan done 1 month after the surgery showed no other sites of disease except the thyroid. No lytic bone lesions were present. The patient was treated with 50.4 Gy of radiation by external beam radiotherapy to the thyroid in 28 fractions as definitive therapy. Despite treatment with surgery and radiation, she continued to have pain around the neck, and a repeat PET scan 3 months after completion of radiation showed persistent uptake in the thyroid. Because of her refractoriness to radiotherapy, she was started on systemic therapy with a weekly regimen of bortezomib and dexamethasone for 9 cycles. Her symptoms began to resolve, and a repeat PET scan done after completion of chemotherapy showed no evidence of uptake, suggesting adequate response to chemotherapy, and chemotherapy was therefore stopped. The patient was scheduled a regular follow-up in 3 months. 
Because of continued local symptoms in this follow-up period, the decision was made to perform surgical gland removal, and she underwent completion of thyroidectomy by otorhinolaryngology.

\section{Discussion}

Plasma cells are well-differentiated B-lymphocytes that secrete antibodies and provide protective immunity to the human body. ${ }^{9}$ Plasma cell neoplasms are clonal proliferation of plasma cells, producing monoclonal immunoglobulins. They are of the following different types: plasma cell myeloma, monoclonal gammopathy of unknown significance, immunoglobulin deposition disease, POEMS (polyneuropathy, organomegaly, endocrinopathy, monoclonal protein, skin changes) syndrome, and plasmacytomas, which are divided into 2 types - solitary plasmacytoma of the bone, and extramedullary plasmacytoma (EMP). ${ }^{10} \mathrm{EMP}$ is a rare condition and encompasses $3 \%$ to $5 \%$ of all plasma cell neoplasms, depending on the study. ${ }^{1,2,5}$ It is more common in men than in women (2.6:1, respectively), with equal incidence among black and white patients. Median age at diagnosis is 62 years, and it is more common among those aged 40 to 70 years. ${ }^{2,11}$ The most common sites of occurrence are the respiratory tract, the mouth, and the pharynx, but other sites such as the eyes, brain, skin, and lymph nodes may also be involved. ${ }^{2}$

EMP involving the thyroid gland is a very rare occurrence, but plasma cell myeloma has been shown to secondarily involve the thyroid. ${ }^{4}$ Similar to our report, EMP of the thyroid in the setting of thyroiditis has been reported by other authors. ${ }^{3,4}$ The incidence of EMP occurring in the thyroid varies according to different authors. Wiltshaw found 7 cases involving the thyroid out of 272 cases of EMP. ${ }^{1}$ Galieni and colleagues reported only 1 case that involved the thyroid out of 46 described cases of EMP. ${ }^{12}$

El- Siemińska and colleagues showed that levels of interleukin (IL)-6 are elevated in thyroiditis. ${ }^{13}$ IL-6 promotes monoclonal as well as polyclonal proliferation of plasma cells. Kovalchuk and colleagues showed an increase in EMP in IL-6 transgenic mice, suggesting a pathophysiologic explanation. ${ }^{14}$

The diagnostic requirements of EMP include the following: histology showing monoclonal plasma cell infiltration in tissue; bone marrow biopsy with normal plasma cell aspirate and biopsy (plasma cells, $<5 \%$ ); no lytic lesions on skeletal survey; no anemia, renal impairment, or hypercalcemia; and absent or low serum M protein. ${ }^{12}$

Our case fulfilled those criteria.

The treatment options for EMP include surgery, radiotherapy, or a combined approach including both. Usually, EMPs are very sensitive to radiotherapy, and complete remission can be achieved by radiotherapy alone in $80 \%$ to $100 \%$ of cases. ${ }^{6,11,15}$ Surgery is considered if the tumor is diffuse or is causing symptoms secondary to pressure on surrounding structures. A combined approach is recommended in cases with incomplete surgical margin or lymph node involvement. ${ }^{5,6}$

There is limited evidence about and experience with the use of chemotherapy in the treatment of EMP. It has been recommended that chemotherapy be considered in patients with refractory or relapsed disease using the same regimen used in plasma cell myeloma. ${ }^{5}$ Katodritou and colleagues have reported using bortezomib and dexamethasone without surgery in a solitary gastric plasmacytoma to avoid the toxicity of gastrointestinal irradiation. ${ }^{7}$ Wei and colleagues treated a patient with EMP in the pancreas with bortezomib and achieved a near-complete remission. ${ }^{8}$ To our knowledge, there is no documented literature about the treatment of EMP of the thyroid with chemotherapy. In our patient, despite the treatment with surgery and radiation, there was persistent uptake on the PET scan, so we treated her with bortezomib and dexamethasone. Because there is an $11 \%$ to $30 \%$ risk of progression to multiple myeloma, long-term follow-up is recommended in EMP. ${ }^{11}$

\section{Conclusions}

Solitary EMP of the thyroid gland is a rare condition. Plasma cell myeloma must be ruled out to make a diagnosis. Data on the incidence of EMP and its clinicopathological features are sparse, and literature describing proper guidelines on treatment is limited. It can be treated with radiotherapy, surgery, or a combined approach. There is limited data on the role of chemotherapy; our case adds to the available literature on using myeloma-based therapy in refractory disease and, to our knowledge, is the only case report using this in the literature on cases of EMP of the thyroid. Regular follow-up, even after the disease is in remission, is necessary because of the high risk of progression to plasma cell myeloma.

\section{References}

1. Wiltshaw E. The natural history of extramedullary plasmacytoma and its relation to solitary myeloma of bone and myelomatosis. Medicine (Baltimore). 1976;55(3):217-238.

2. Dores GM, Landgren O, McGlynn KA, Curtis RE, Linet MS, Devesa SS. Plasmacytoma of bone, extramedullary plasmacytoma, and multiple myeloma: incidence and survival in the United States, 1992-2004. Br J Haematol. 2009;144(1):86-94.

3. Kovacs CS, Mant MJ, Nguyen GK, Ginsberg J. Plasma cell lesions

of the thyroid: report of a case of solitary plasmacytoma and a review of the literature. Thyroid. 1994;4(1):65-71.

4. Avila A, Villalpando A, Montoya G, Luna MA. Clinical features and differential diagnoses of solitary extramedullary plasmacytoma of the thyroid: a case report. Ann Diagn Pathol. 2009;13(2):119-123.

5. Hughes M, Soutar R, Lucraft H, Owen R, Bird J. Guidelines on the diagnosis and management of solitary plasmacytoma of bone, extramedullary plasmacytoma and multiple solitary plasmacytomas: 2009 
update. London, United Kingdom: British Committee for Standards in Haematology; 2009.

6. Weber DM. Solitary bone and extramedullary plasmacytoma. Hematology Am Soc Hematol Educ Program. 2005;373-376.

7. Katodritou E, Kartsios C, Gastari V, et al. Successful treatment of extramedullary gastric plasmacytoma with the combination of bortezomib and dexamethasone: first reported case. Leuk Res. 2008;32(2):339-341.

8. Wei JY, Tong HY, Zhu WF, et al. Bortezomib in treatment of extramedullary plasmacytoma of the pancreas. Hepatobiliary Pancreat Dis Int. 2009;8(3):329-331.

9. Roth K, Oehme L, Zehentmeier S, Zhang Y, Niesner R, Hauser AE. Tracking plasma cell differentiation and survival. Cytometry A. 2014;85(1):15-24.

10. Swerdlow SH, Campo E, Harris NL, et al. WHO classification of tumours of haematopoietic and lymphoid tissues. 4 th ed. Lyon,
France: International Agency for Research on Cancer; 2008.

11. Alexiou C, Kau RJ, Dietzfelbinger H, et al. Extramedullary plasmacytoma: tumor occurrence and therapeutic concepts. Cancer. 1999;85(11):2305-2314.

12. Galieni P, Cavo M, Pulsoni A, et al. Clinical outcome of extramedullary plasmacytoma. Haematologica. 2000;85(1):47-51.

13. Siemińska L, Wojciechowska C, Kos-Kudła B, et al. Serum concentrations of leptin, adiponectin, and interleukin-6 in postmenopausal women with Hashimoto's thyroiditis. Endokrynol Pol. 2010;61(1):112-116.

14. Kovalchuk AL, Kim JS, Park SS, et al. IL-6 transgenic mouse model for extraosseous plasmacytoma. Proc Natl Acad Sci USA. 2002;99(3):1509-1514.

15. Chao MW, Gibbs P, Wirth A, Quong G, Guiney MJ, Liew KH. Radiotherapy in the management of solitary extramedullary plasmacytoma. Intern Med J. 2005;35(4):211-215. 